Open Access

\title{
Porcine epidemic diarrhea virus inhibits dsRNA-induced interferon- $\beta$ production in porcine intestinal epithelial cells by blockade of the RIG-I-mediated pathway
}

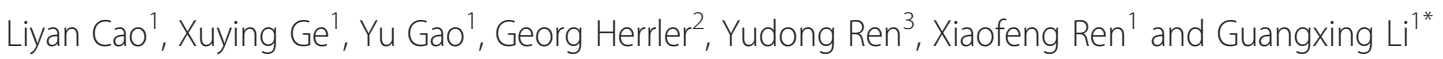

\begin{abstract}
Background: The lack of optimal porcine cell lines has severely impeded the study and progress in elucidation of porcine epidemic diarrhea virus (PEDV) pathogenesis. Vero cell, an African green monkey kidney cell line, was often used to isolate and propagate PEDV. Nonetheless, the target cells of PEDV in vivo are intestinal epithelial cells, during infection, intestinal epithelia would be damaged and resulted in digestive disorders. The immune functions of porcine epithelial cells and interactions with other immune cell populations display a number of differences compared to other species. Type I interferon (IFN) plays an important role in antiviral immune response. Limited reports showed that PEDV could inhibit type I interferon production. In this study, porcine small intestinal epithelial cells (IECs), the target cells of PEDV, were used as the infection model in vitro to identify the possible molecular mechanisms of PEDV-inhibition IFN- $\beta$ production.
\end{abstract}

Results: PEDV not only failed to induce IFN- $\beta$ expression, but also inhibited dsRNA-mediated IFN- $\beta$ production in IECs. As the key IFN- $\beta$ transcription factors, we found that dsRNA-induced activation of IFN regulatory factor 3 (IRF-3) was inhibited after PEDV infection, but not nuclear factor-kappaB (NF-kB). To identify the mechanism of PEDV intervention with dsRNA-mediated IFN- $\beta$ expression more accurately, the role of individual molecules of RIG-I signaling pathway were investigated. In the upstream of IRF-3, TANK-binding kinase 1 (TBK1)-or inhibitor of $\mathrm{KB}$ kinase- $\varepsilon$ (IKKE)-mediated IFN- $\beta$ production was not blocked by PEDV, while RIG-I-and its adapter molecule IFN- $\beta$ promoter stimulator 1 (IPS-1)-mediated IFN- $\beta$ production were completely inhibited after PEDV infection.

Conclusion: Taken together, our data demonstrated for the first time that PEDV infection of its target cell line, IECs, inhibited dsRNA-mediated IFN- $\beta$ production by blocking the activation of IPS-1 in RIG-I-mediated pathway. Our studies offered new visions in understanding of the interaction between PEDV and host innate immune system.

\section{Background}

Porcine epidemic diarrhea virus (PEDV) is an enveloped, single-stranded, RNA virus of Coronaviridae family, which is the main etiological agent of severe diarrhea in pigs of all ages and fatality in neonates [1]. Outbreaks of porcine epidemic diarrhea (PED) have received extensive attention for the considerable economic losses to the swine industry worldwide. Great advances have been made in elucidation of the molecular epidemiology, diagnosis, prevention, and

\footnotetext{
*Correspondence: ligx@neau.edu.cn

${ }^{1}$ College of Veterinary Medicine, Northeast Agricultural University, 59 Mucai Street, Xiangfang District, Harbin 150030, China

Full list of author information is available at the end of the article
}

treatment of PED [2]. Recently, coronavirus interaction with host innate immune system has been a hot research field. Previous studies indicated that transmissible gastroenteritis virus (TGEV) infection enhanced type I interferon expression and its protein 7 modulated type I IFN expression [3, 4]. For mouse hepatitis virus (MHV), IFN production among different cell populations varied due to their diverse susceptibility to this virus [5-9]. Furthermore, both severe acute respiratory syndrome coronavirus (SARS-CoV) and Middle East respiratory syndrome coronavirus (MERS-CoV) do not induce type I IFN (IFN- $\alpha$ / $\beta)$ activation [10-12]. So far, limited reports showed that PEDV could inhibit type I interferon production [13, 14]. 
During viral infection and replication, the host innate immune response is the first line of defense; therefore, the ability of viruses to suppress or avoid this response is crucial for their pathogenic potential. IFN- $\alpha / \beta$ is an essential element of the host innate immune response against viral infections. Double-stranded RNA (dsRNA), the replicative intermediate of most viruses, is a potent inducer of IFN- $\beta$, which is recognized as a pathogen-associated molecular pattern (PAMP) by host pattern recognition receptors (PRRs). Two of major PRRs, retinoic acid-inducible gene I (RIG-I) and melanoma differentiation-associated gene 5 (MDA5) detect dsRNA in the cytoplasm [15]. Following dsRNA binding, RIG-I and MDA5 recruit corresponding adapter protein IFN- $\beta$ promoter stimulator 1 (IPS-1) that, in turn, activate downstream signaling of TANK-binding kinase 1 (TBK1) and inhibitor of $\kappa B$ kinase- $\varepsilon$ (IKK $\varepsilon$ ) transduction, leading to the activation of transcription factor IFN regulatory factor 3 (IRF-3) and nuclear factor-kappaB $(\mathrm{NF}-\mathrm{kB})$. Activated IRF-3, and NF-kB bind to IFN- $\beta$ enhancer and initiate IFN- $\beta$ transcription [16].

Vero cell, an African green monkey kidney cell line, was often used to isolate and propagate PEDV [17]. However, it was often considered that Vero cells might lack genetic component necessary for IFN production [18-20]. Porcine intestinal epithelial cells (IECs) are thought to the target cells of PEDV, which play an important role in the activation of host immune responses by induction of key signaling molecules, including cytokines, surface molecules, and chemokines during microoganism invasion $[21,22]$. In the present study, to determine if PEDV infection suppresses IFN- $\beta$ activation, we chose IECs as an infection model to research the molecular mechanisms of PEDV infection and the host antiviral innate immune response. Our results clearly suggested that PEDV prevented dsRNA-induced IFN- $\beta$ synthesis by blocking RIG-I-mediated pathways.

\section{Results and discussion}

PEDV failed to induce IFN- $\beta$ expression and inhibited poly (I:C)-mediated IFN- $\beta$ production in IECs

Type I IFNs $($ IFN- $\alpha / \beta)$ are critical to the host antiviral innate immune response. However, there is no evidence suggesting that IECs produce type I IFNs in response to PEDV infection. Previous studies have showed that PEDV could be propagated in IECs [ 23, 24,]. To confirm whether PEDV infection could induce IFN- $\beta$ production in IECs or not, we transiently cotransfected the IFN- $\beta$ / luciferase reporter plasmid (IFN- $\beta$-Luc) and the Renilla luciferase construct phRL-TK and then infected with PEDV (at an MOI of 1 or 0.1 , respectively) or mockinfected for $24 \mathrm{~h}$. The cells were retransfected with $1 \mu \mathrm{g}$ of poly ( $\mathrm{I}: \mathrm{C})$ as a positive inducer. As shown in Fig. 1a, IFN- $\beta$ luciferase activity enhanced markedly in positive controls, while it was almost not detected in
PEDV-infected IECs. In addition, IFN- $\beta$ mRNA expression was hardly detected in PEDV-infected IECs similar to mock-infected group, however, it had significant expression in poly (I:C)-transfected group at the indicated times (12 $\mathrm{h}$ and $24 \mathrm{~h}, \mathrm{p}<0.01$ ) (Fig. 1b). This result was consistent with the luciferase reporter assay. Taken together, PEDV infection of IECs did not induce IFN- $\beta$ activation.

Increasing evidence showed that viruses not only inhibit the induction of type I IFNs, but also block dsRNAinduced production of type I IFNs to escape the innate immune surveillance of the host [25-27]. To identify whether PEDV was able to inhibit dsRNA-induced IFN- $\beta$ production, IFN- $\beta$-Luc was transfected into PEDV-infected and uninfected cells, respectively. The cells were retransfected with or without poly (I:C) $24 \mathrm{~h}$ later. As a result, activation of the IFN- $\beta$ promoter decreased significantly in poly (I:C)-transfected, PEDVinfected cells compared with mock-infected cells transfected with poly (I:C) (Fig. 1c). It showed PEDV also inhibited poly (I:C)-mediated IFN- $\beta$ induction.

\section{PEDV impeded poly (I:C)-mediated activation of IRF-3, but not NF-KB}

IRF-3 and NF- $\mathrm{BB}$ are two essential IFN- $\beta$ transcription factors. In unstimulated cells, IRF-3 is ubiquitously present in the cytoplasm as an inactive monomer, whereas NF- $\mathrm{kB}$ is present as a homodimer or heterodimer bound to the inhibitory proteins IкB in the cytoplasm [28]. Phosphorylation, which is a key step during IRF-3 and NF$\kappa B$ activation, in turn, leads to nuclear translocation. Therefore, we evaluated whether PEDV infection induced IRF-3 and p65 activation by western blot analysis. Following PEDV infection, the whole cell extracts were prepared for the indicative times, in Fig. 2a, IRF-3 still existed in the cytoplasm, and the levels of IRF-3 protein was almost equal with mock-infected cells, while the phosphorylation of IRF3 (p-IRF-3) did not detect in the PEDV infected cells in comparision to a obviously signal in poly (I:C)-transfected cells. On the contrary, compared with the amount of NF$\mathrm{kB}$ subunit p65 in the cytoplasm, the phosphorylation of p65 (p-p65) increased with progression of PEDV infection in the nucleus. Meanwhile, the concentration of poly (I:C)induced p65 nuclear translocation was clearly increased. PEDV N protein was also detected in PEDV-infected cells. These data suggested that PEDV did not induce activation of IRF-3, but NF-kB.

We then used luciferase reporter assay system to determine whether IRF-3 and NF- $\mathrm{kB}$ are linked with the inhibition of IFN- $\beta$ production after PEDV infection. As shown in Fig. 2b, IRF-3 luciferase activity was sharply decreased in PEDV-infected cells, and poly (I:C)-induced IRF-3 activation was also inhibited by PEDV in comparison to a remarkably signal in poly (I:C)-transfected cells. However, in Fig. 2c, compared with mock-infected 
A

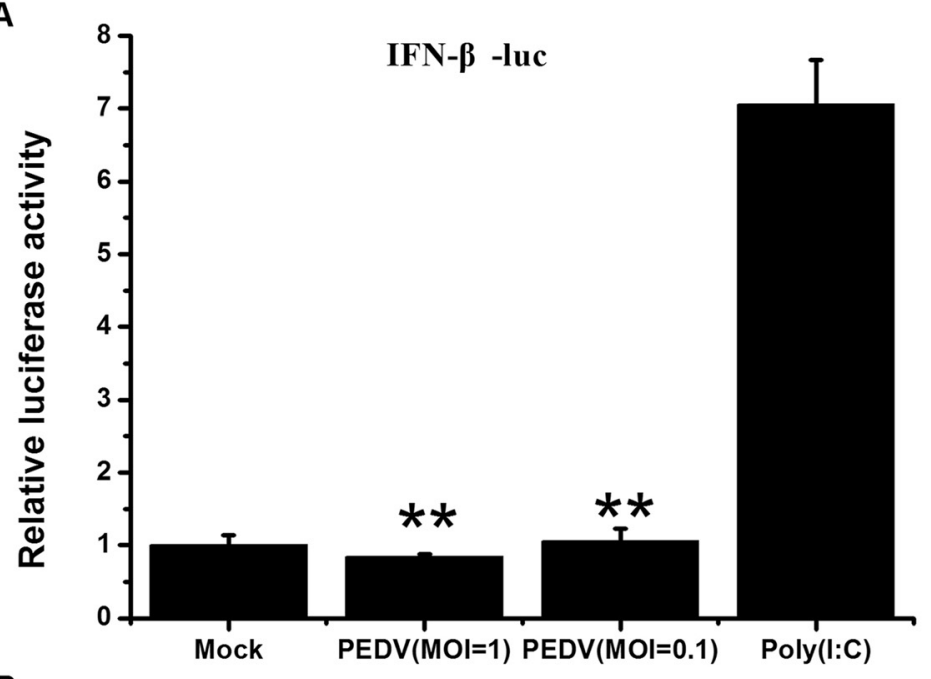

B

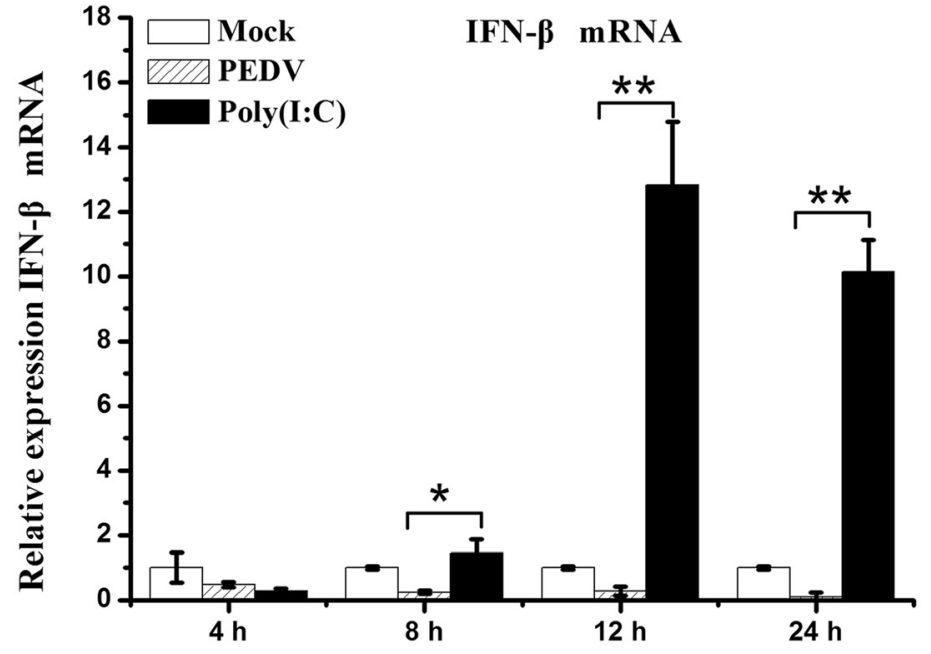

C

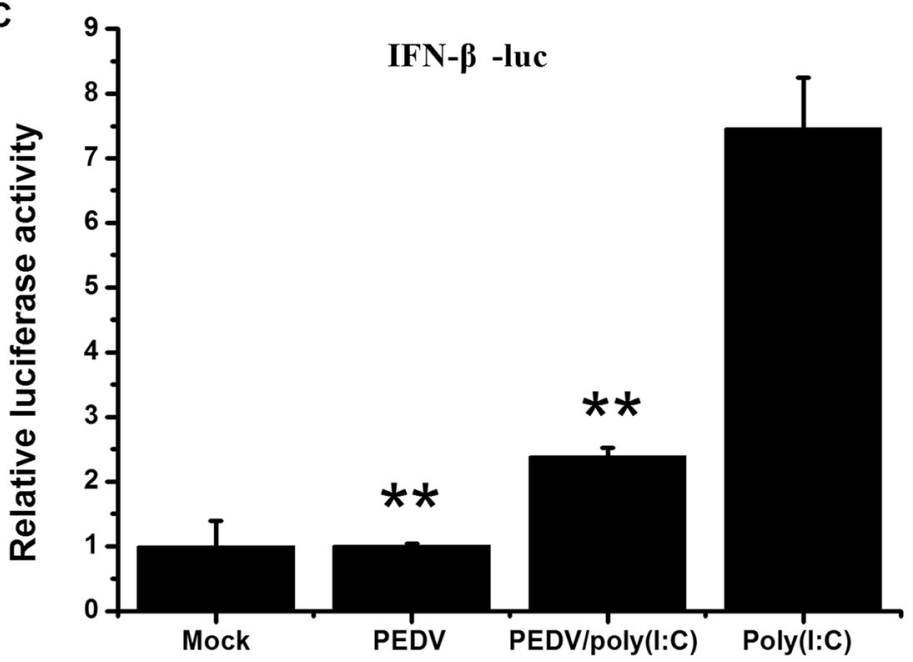

Fig. 1 (See legend on next page.) 
(See figure on previous page.)

Fig. 1 PEDV does not induce IFN- $\beta$ production and inhibits poly (I:C)-mediated IFN- $\beta$ induction. a IECs were cotransfected with IFN- $\beta$-LuC and phRL-TK, then infected with PEDV at an MOI of 1 and 0.1 for $24 \mathrm{~h}$. Cells were retransfected with poly (I:C) as a positive control. After $12 \mathrm{~h}$, the cells were harvested and subjected to a dual-luciferase assay. $\mathbf{b}$ IECs were infected with PEDV at an $\mathrm{MOI}$ of 1 , mock-infected as a negative control, or transfected with poly (I:C) as a positive control. At the indicated time points, total RNA was extracted and IFN- $\beta$ and $\beta$-actin mRNA were subjected to real-time PCR. RNA expression levels were normalized to $\beta$-actin. $\mathbf{c}$ In contrast to a, IECs were first mock-infected or infected with PEDV at an $\mathrm{MOI}$ of $1 \mathrm{for} 12 \mathrm{~h}$ and then cotransfected with IFN- $\beta$-Luc and phRL-TK for $24 \mathrm{~h}$. Cells were retransfected with or without poly (I:C) for an addition $12 \mathrm{~h}$, harvested, and then subjected to a dual-luciferase assay. All data are expressed as means \pm SD of 3 independent experiments. ${ }^{*} \mathrm{p}<0.05$; ${ }^{* *} \mathrm{p}<0.01$ as compared with poly $(\mathrm{I}: \mathrm{C})$

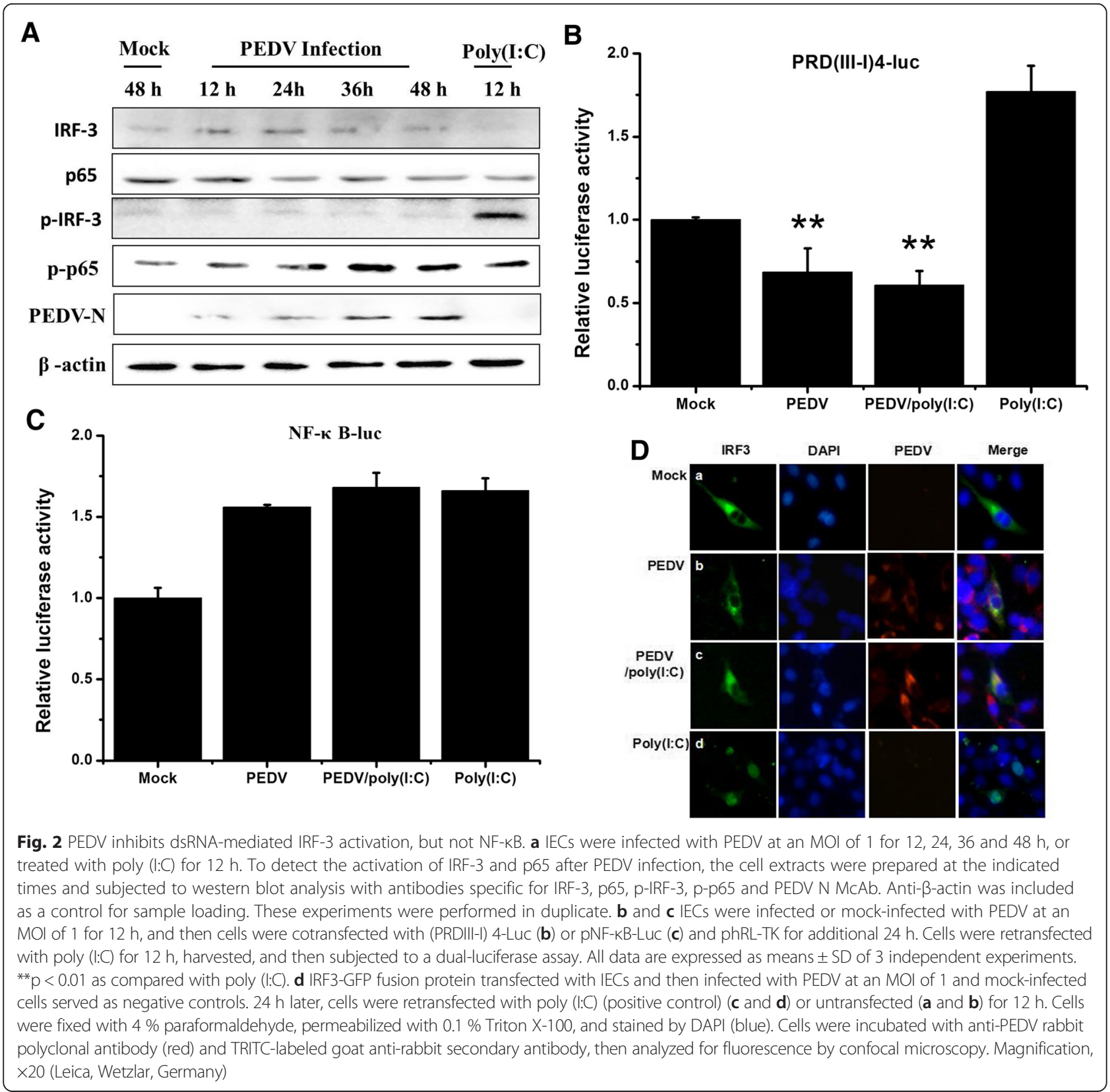


cells, NF-kB luciferase activity significantly enhanced both in PEDV-infected and poly (I:C)-transfected cells. In addition, we found that poly (I:C)-induced activation of NF-kB was not blocked by PEDV. To further identify PEDV-inhibited poly (I:C)-mediated activation of IRF-3, confocal microscopy assay was used. As a result, IRF3-GFP remained in the cytoplasm of both mockinfected (Fig. 2d. a) and PEDV-infected (Fig. 2d. b) IECs compared with poly (I:C) controls, in which clear translocation to the nucleus was observed (Fig. 2d. d). Furthermore, PEDV could block poly (I:C)-mediated IRF-3 nucleus migration (Fig. 2d. c). Taken together, our date clearly implied that PEDV impeded dsRNA-mediated IFN- $\beta$ transcription primary by interfering with IRF-3 activation, but not NF- $\mathrm{kB}$.

\section{PEDV failed to block TBK1/IKKe activity}

TBK1 and IKK $\varepsilon$ are essential kinases for the IRF-3 activation [29]. In order to ascertain whether PEDV inhibited poly (I:C)-induced IRF-3 activation by impeding TBK1/IKKe kinase activity, we cotransfected plasmids expressing TBK1/IKK $\varepsilon$ kinase and a plasmid encoding the IFN- $\beta$ promoter of the luciferase reporter into infected or mock-infected IECs and retransfected the cells with or without poly (I:C) at 36 h.p.i. As show in Fig. 3, TBK1/IKKE overexpression increased IFN- $\beta$ promoter activity in both infected and mock-infected IECs, and obviously upregulation was detected in IECs

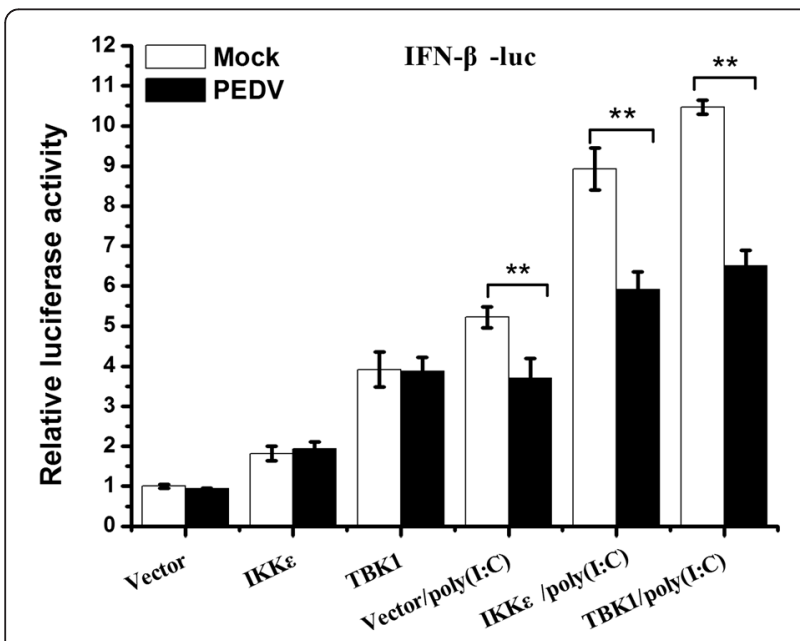

Fig. 3 PEDV does not block the activity of TBK1/IKKE. IECs were infected or mock-infected with PEDV at an MOI of 1 for $12 \mathrm{~h}$, and then cells were cotransfected with the IFN- $\beta$ promoter luciferase reporter and TBK1/IKKE expression plasmids or vector for $24 \mathrm{~h}$. Cells were then retransfected with poly $(\mathrm{I}: \mathrm{C})$ as a positive control for $12 \mathrm{~h}$. Cells were harvested and subjected to a dual-luciferase assay. Data were analyzed and the ratio of firefly luciferase expression to Renilla luciferase activity was shown. All data are expressed as means \pm SD of 3 independent experiments. ${ }^{* *} p<0.01$ compared with PEDV-infected, expression plasmids or vector-transfected control transfected with poly (I:C), suggesting that PEDV failed to block TBK1/IKKe activity. However, poly (I:C)-induced IFN- $\beta$ promoter activity in IECs overexpression of TBK1/ IKK $\varepsilon$ plasmids was significantly inhibited by PEDV. It showed that PEDV interrupting dsRNA-induced IFN- $\beta$ production should localize upstream from TBK1/IKKe.

\section{PEDV inhibited RIG-I-mediated IFN- $\beta$ production}

It is possible that PEDV blocks poly (I:C)-mediated IFN$\beta$ production by suppression of the individual molecules upstream of TBK1/IKKe in RIG-I signaling pathway. To explore this possibility, mock- and PEDV-infected IECs were cotransfected with IPS-1 expression plasmid and IFN- $\beta$ promoter luciferase reporter plasmid, respectively. As shown in Fig. 4a, overexpression of IPS-1 in IECs could enhance IFN- $\beta$ luciferase activity in mock-infected cells, but it was completely inhibited in PEDV-infected cells. For the poly (I:C) transfection experiments, there appeared significant restriction of IFN- $\beta$ luciferase expression in PEDV-infected cells compared with that of mock-infected cells. These date indicated that PEDV interacted with IPS-1 to block poly (I:C)-mediated IFN- $\beta$ transcription.

IPS-1 is an adapter molecule of RIG-I, the data showed that PEDV blocked IPS-1-induced IFN- $\beta$ production in dsRNA signaling pathway, thus, we speculated that RIG-I-induced IFN- $\beta$ production in this signaling pathway was also inhibited. To verify it, mock- and PEDVinfected IECs were transfected with RIG-I expression and IFN- $\beta$ reporter plasmids. The results showed that IFN- $\beta$ luciferase activity was markedly increased in IECs overexpressing RIG-I, but was completely inhibited by PEDV infection. And the IFN- $\beta$ reporter signal could be observed in IECs stimulated with poly (I:C), while this signal could be sharply reduced in RIG-Itransfected, poly (I:C)-stimulated and PEDV-infected IECs (Fig. 4b).

\section{Conclusions}

In summary, the findings of the present study suggested that PEDV-infection in IECs inhibits dsRNA-induced IFN- $\beta$ induction by interfering with IRF-3 activity associated with RIG-I-mediated signaling pathway. The target interaction molecules of PEDV intervention of dsRNAinduced IFN- $\beta$ production primarily was IPS-1. However, as a limitation to this study, host cells may inhibit or activate multiple signaling pathways simultaneously in response to exogenous stimulus, and some other transcription factors may have been blocked or activated in this process. Here, we only addressed the mechanisms of PEDV-induced inhibition of IFN- $\beta$ production in relation to the molecules of RIG-I signaling pathways in vitro. Further studies are needed. Overall, elucidation of the 


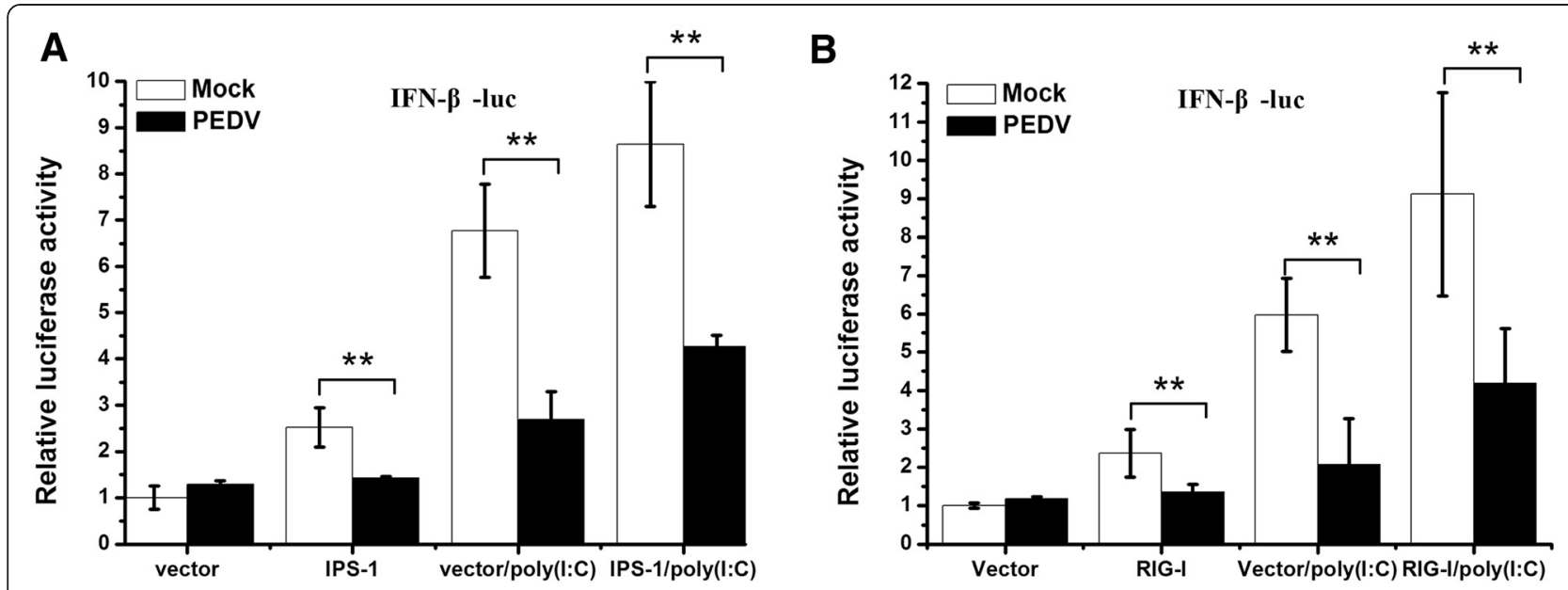

Fig. 4 PEDV inhibits IPS-1- and RIG-I-induced IFN- $\beta$ production. PEDV-infected IECs were cotransfected with the IFN- $\beta$ promoter luciferase reporter and IPS-1 (a) or RIG-I (b) expression plasmids or vector for $24 \mathrm{~h}$. Cells were then retransfected with poly (I:C) for $12 \mathrm{~h}$. Cells were harvested and luciferase activity was analyzed using a dual-luciferase assay. All data are expressed as means \pm SD of 3 independent experiments. ${ }^{* *} p<0.01$ compared with PEDV-infected, expression plasmids or vector-transfected control

influence of PEDV evasion of the host innate immune response will aid in the development of antiviral agents to prevent the spread of PEDV during the early infection phase.

\section{Materials and methods}

\section{Viruses, cells, and reagents}

The African green monkey kidney cell line VeroE6 and swine small intestine epithelial cells (IECs) $[30,31]$ were respectively cultured in Dulbecco's modified Eagle's medium (DMEM) and Dulbecco's modified Eagle's F12 Ham medium (DMEM-F12) supplemented with $10 \%$ fetal bovine serum at $37{ }^{\circ} \mathrm{C}$ in a humidified atmosphere of $5 \% \mathrm{CO}_{2}$. PEDV strain CV777 was propagated in VeroE6 cells in DMEM containing $2.5 \mu \mathrm{g} / \mathrm{mL}$ of trypsin.

Poly (I:C) was purchased as a sodium salt (SigmaAlorch, Saint Louis, MO, USA) and dissolved in water to obtain a stock solution of $10 \mathrm{mg} / \mathrm{mL}$. The DualLuciferase $^{\circ}$ Reporter Assay System was purchased from Promega Corporation (Madison, WI, USA) and monoclonal anti- $\beta$-actin antibody was purchased from SigmaAldrich (St. Louis, MO, USA). Anti-IRF-3, anti-p65 anti-p-IRF-3 and anti-p-p65 rabbit polyclonal antibodies and secondary horseradish peroxidase (HRP)-conjugated anti-rabbit IgG were purchased from Cell Signaling Technology, Inc. (Beverly, MA, USA). Rhodamine isothiocyanate (TRITC)-labeled goat anti-rabbit IgG were purchased from the Zhongshan Company (Beijing, China). Anti-PEDV rabbit polyclonal antibodies and anti-PEDV N protein monoclonal antibody (McAb) were prepared in our laboratory, which could specifically react with PEDV.

\section{Plasmids}

The plasmids IFN- $\beta$-Luc for IFN- $\beta$, PRD (III-I) 4-Luc for IRF-3, and pNF-kB-Luc for NF- $\kappa$ B were kindly donated by Dr. Shaobo Xiao (Huazhong Agricultural University, Wuhan, Hubei Province, China) [25]. The pEF-BOS empty vector and pEF-Flag-RIG-I recombinant expression plasmid were kindly provided by $\mathrm{T}$. Fujita (Tokyo Metropolitan Institute of Medical Science, Tokyo, Japan) [32]. The pEF-Bos-Flag-TRIF, pCDNA3-Flag-IKKe and pCDNA3-Flag-TBK1 recombinant expression plasmids, and the pCDNA3 empty vector, and the conjugate IRF3-green fluorescence protein (GFP) expression construct were kindly provided by K. Fitzgerald (University of Massachusetts Medical School, Worcester, MA, USA) [27]. The porcine IPS-1 (NCBI accession No: EU082069.1) gene was cloned from porcine kidney cells by reverse transcription polymerase chain reaction (RT-PCR) using the specific primer pair. The IPS-1 primers were $5^{\prime}$ CCGGGTACCACCATGACGTTTGCCGAGGACAA-3' and 5 '-TTTCTCGAGTCACTGGGGCAGGCGCCGCC-3' . Porcine IPS-1 was inserted into pcDNA3.1 (+) using the restriction enzymes KpnI and XhoI.

\section{Transfection and luciferase assay}

IECs were plated in 24-well plates at a density of $1 \times 10^{5}$ cells/well and cotransfected with $0.2 \mu \mathrm{g}$ of the luciferase reporter plasmids (IFN- $\beta$-Luc, [PRDIII-I] 4-Luc, and pNF- $\kappa$ B-Luc, respectively), and the Renilla luciferase construct phRL-TK (Promega Corp.), as an internal control $(0.1 \mu \mathrm{g})$ with Lipofectamine 2000 reagent (Invitrogen Corp.) according to the manufacturer's instructions 
when the cells reached $70 \%-80 \%$ confluence. The cells were then infected or mock-infected with PEDV for $24 \mathrm{~h}$. Cells were retransfected with or without poly (I:C) $(1.0 \mu \mathrm{g})$ for an additional $12 \mathrm{~h}$. Or IECs were infected or mock-infected with PEDV for $12 \mathrm{~h}$ prior to transfection the luciferase reporter plasmids alone or cotransfection the indicated expression plasmids $(0.5 \mu \mathrm{g})$. The cell lysates were harvested and luciferase activity was analyzed using a dual-luciferase assay system and a luminometer (Turner BioSystems, Inc. Sunnyvale, CA, USA) according to the manufacturer's instructions. Data represent relative firefly luciferase activity normalized to Renilla luciferase activity. The resulting ratios were used to compare the expression of the firefly luciferase gene in PEDVinfected cells to that present in mock-infected cells.

\section{Real-time PCR amplification of IFN- $\beta$}

Total RNA was extracted from the transfected cells using TriQuick reagent (Beijing Solarbio Science \& Technology Co., Ltd., Beijing, China) according to the manufacturer's instructions and then reverse-transcribed into complementary DNA (cDNA) using murine leukemia virus reverse transcriptase (GBI Labs/Golden Bridge International, Inc., Mukilteo, WA, USA) with oligo dT random hexamers (HaiGene Technology, Harbin, China). The cDNA was then subjected to real-time PCR with specific primer pairs targeting IFN- $\beta$ (F: 5 '-GCTAAC AAGTGCATCCTCCAAA-3' and R: 5'-CCAGGAGC TTCTGACATGCCA-3') and $\beta$-actin (F: $5^{\prime}$ - GGCTCAG AGCAAGAGAGGTATCC-3', and R: $5^{\prime}$-GGTCTCAAA CATGATCTGAGTCATCT-3'. $\beta$-actin mRNA was used as an endogenous control.

\section{Nuclear translocation assay}

IECs were seeded in 24-well plates and then transfected with $1 \mu \mathrm{g}$ of IRF3-GFP fusion expression constructs per well using Lipofectin transfection reagent (Invitrogen Corp.) when cells reached confluence. Cells were then mock-infected or infected with PEDV at a multiplicity of infection (MOI) of 1 . At $24 \mathrm{~h}$ postinfection, cells were transfected with $1 \mu \mathrm{g}$ of poly (I:C) or left untransfected. After $12 \mathrm{~h}$, cells were removed from the culture medium and washed three times in cold phosphatebuffered saline (PBS). Next, cells were fixed in $4 \%$ paraformaldehyde for $15 \mathrm{~min}$ at room temperature, quenched with $0.1 \mathrm{M}$ glycine for $5 \mathrm{~min}$, and then permeabilized with $0.1 \%$ Triton X-100 for $10 \mathrm{~min}$. Afterward, the cells were incubated with anti-PEDV antibody (dilution, 1:500) for $1 \mathrm{~h}$ followed by TRITClabeled goat anti-rabbit secondary antibody (dilution, 1:200) for $30 \mathrm{~min}$ at $37^{\circ} \mathrm{C}$. The nuclei were stained with 4',6-diamidino-2-phenylindole-dihydrochloride (DAPI) (Invitrogen Corp.). Cells were examined using a TCS
SP2 AOBS confocal microscope (Leica Camera AG, Wetzlar, Germany).

\section{Western blot analysis}

IECs were infected with PEDV at an MOI of 1 or treated with poly (I:C) for the indicative times, lysed in $2 \times$ sodium dodecyl sulfate (SDS) sample buffer and boiled for 10 min. Whole Cells extracts were separated by $12 \%$ SDS-polyacrylamide gel electrophoresis and transferred to a nitrocellulose membrane, which was blocked with $5 \%(\mathrm{w} / \mathrm{v})$ bovine serum albumin (BSA) in tris-buffered saline $(10 \mathrm{mM}$ Tris- $\mathrm{Cl}$ at $\mathrm{pH} 7.5$ and $150 \mathrm{mM} \mathrm{NaCl})$ containing $0.05 \%$ Tween 20 (TBST) at room temperature for $1 \mathrm{~h}$. The membranes were then incubated with a primary antibody (dilution, 1:1000) at $4{ }^{\circ} \mathrm{C}$ overnight and a secondary HRP-conjugated antibody (dilution, 1:2500) for $1 \mathrm{~h}$ at room temperature. Protein blots were developed using an enhanced chemiluminescence (ECL) detection system and exposed to X-ray film (Clinx Science Instruments Co., Ltd., Shanghai, China).

\section{Statistical analysis}

All data were expressed as means \pm standard deviations (SD) of 3 independent experiments. The statistical significance was tested by student 's t-test and p-values less than 0.05 were considered statistically significant.

\section{Competing interests}

All authors declare that there are no financial or other relationships that might lead to a conflict of interests.

\section{Authors' contributions}

LYC response for carrying out the experiments, date analysis and drafting the manuscript. XYG and YG construct the recombinant expression plasmids. YDR participated in date analysis. GH, XFR and GXL designed the experiments and reviewed manuscript. All authors have seen and approved the manuscript and have contributed significantly to the work.

\section{Acknowledgments}

We thank Shaobo Xiao, Takashi Fujita, Kate Fitzgerald for kindly providing important constructs. This work was supported by the programme for New Century Excellent Talents at the Heilongjiang Provincial University (1155-NCET-005); National Natural Science Foundation of China (31270187; 31372438); National Science \& Technology Pillar Program during the Twelfth Five-year Plan Period (2013BAD12B04); and Doctoral Foundation of Ministry of Education of China (20122325110019).

\section{Author details}

${ }^{1}$ College of Veterinary Medicine, Northeast Agricultural University, 59 Mucai Street, Xiangfang District, Harbin 150030, China. ${ }^{2}$ Institute of Virology University of Veterinary Medicine, BÜnteweg 17, D-30559 Hannover, Germany. ${ }^{3}$ College of Electrical and Information, Northeast Agricultural University, Harbin 150030, China.

Received: 20 January 2015 Accepted: 21 July 2015

Published online: 18 August 2015

\section{References}

1. Pijpers A, Van Nieuwstadt AP, Terpstra C, Verheijden JH. Porcine epidemic diarrhoea virus as a cause of persistent diarrhoea in a herd of breeding and finishing pigs. Vet Rec. 1993;132:129-31.

2. Song D, Park B. Porcine epidemic diarrhoea virus: a comprehensive review of molecular epidemiology, diagnosis, and vaccines. Virus Genes. 2012;44:167-75. 
3. Charley B, Laude H. Induction of alpha interferon by transmissible gastroenteritis coronavirus: role of transmembrane glycoprotein E1. J Virol. 1988;62:8-11.

4. Cruz JL, Becares M, Sola I, Oliveros JC, Enjuanes L, Zuniga S. Alphacoronavirus protein 7 modulates host innate immune response. J Virol. 2013;87:9754-67.

5. Garlinghouse Jr LE, Smith AL, Holford T. The biological relationship of mouse hepatitis virus (MHV) strains and interferon: in vitro induction and sensitivities. Arch Virol. 1984;82:19-29.

6. Li J, Liu Y, Zhang X. Murine coronavirus induces type I interferon in oligodendrocytes through recognition by RIG-I and MDA5. J Virol. 2010;84:6472-82.

7. Roth-Cross JK, Martinez-Sobrido L, Scott EP, Garcia-Sastre A, Weiss SR. Inhibition of the alpha/beta interferon response by mouse hepatitis virus at multiple levels. J Virol. 2007;81:7189-99.

8. Zhou H, Perlman S. Preferential infection of mature dendritic cells by the JHM strain of mouse hepatitis virus. Adv Exp Med Biol. 2006;581:411-4.

9. Zhou H, Perlman S. Mouse hepatitis virus does not induce Beta interferon synthesis and does not inhibit its induction by double-stranded RNA. J Virol. 2007:81:568-74.

10. Cheung $\mathrm{CY}$, Poon LL, Ng IH, Luk W, Sia SF, Wu MH, et al. Cytokine responses in severe acute respiratory syndrome coronavirus-infected macrophages in vitro: possible relevance to pathogenesis. J Virol. 2005;79:7819-26.

11. Kindler E, Jonsdottir HR, Muth D, Hamming OJ, Hartmann R, Rodriguez R, et al. Efficient replication of the novel human betacoronavirus EMC on primary human epithelium highlights its zoonotic potential. MBio. 2013:4:e00611-2

12. Ziegler T, Matikainen S, Ronkko E, Osterlund P, Sillanpaa M, Siren J, et al. Severe acute respiratory syndrome coronavirus fails to activate cytokinemediated innate immune responses in cultured human monocyte-derived dendritic cells. J Virol. 2005;79:13800-5.

13. Xing Y, Chen J, Tu J, Zhang B, Chen X, Shi H, et al. The papain-like protease of porcine epidemic diarrhea virus negatively regulates type I interferon pathway by acting as a viral deubiquitinase. J Gen Virol. 2013;94:1554-67.

14. Ding Z, Fang L, Jing H, Zeng S, Wang D, Liu L, et al. Porcine epidemic diarrhea virus nucleocapsid protein antagonizes beta interferon production by sequestering the interaction between IRF3 and TBK1. J Virol. 2014;88:8936-45.

15. Andrejeva J, Childs KS, Young DF, Carlos TS, Stock N, Goodbourn S, et al. The $V$ proteins of paramyxoviruses bind the IFN-inducible RNA helicase, mda-5, and inhibit its activation of the IFN-beta promoter. Proc Natl Acad Sci U S A. 2004;101:17264-9.

16. Thanos D, Maniatis T. Virus induction of human IFN beta gene expression requires the assembly of an enhanceosome. Cell. 1995;83:1091-100.

17. Hofmann M, Wyler R. Propagation of the virus of porcine epidemic diarrhea in cell culture. J Clin Microbiol. 1988;26:2235-9.

18. Desmyter J, Melnick JL, Rawls WE. Defectiveness of interferon production and of rubella virus interference in a line of African green monkey kidney cells (Vero). J Virol. 1968;2:955-61.

19. Emeny JM, Morgan MJ. Regulation of the interferon system: evidence that Vero cells have a genetic defect in interferon production. J Gen Virol. 1979;43:247-52.

20. Frieman M, Heise M, Baric R. SARS coronavirus and innate immunity. Virus Res. 2008;133:101-12.

21. Pitman RS, Blumberg RS. First line of defense: the role of the intestinal epithelium as an active component of the mucosal immune system. J Gastroenterol. 2000;35:805-14.

22. Schierack P, Nordhoff M, Pollmann M, Weyrauch KD, Amasheh S, Lodemann $U$, et al. Characterization of a porcine intestinal epithelial cell line for in vitro studies of microbial pathogenesis in swine. Histochem Cell Biol. 2006;125:293-305.

23. Cao L, Ge X, Gao Y, Ren Y, Ren X, Li G: Porcine epidemic diarrhea virus infection induces NF-kappaB activation through the TLR2, TLR3, and TLR9 pathways in porcine intestinal epithelial cells. J Gen Virol 2015. doi: 10.1099/ vir.0.000133.

24. Cong Y, Li X, Bai Y, LV X, Herrler G, Enjuanes L, et al. Porcine aminopeptidase $\mathrm{N}$ mediated polarized infection by porcine epidemic diarrhea virus in target cells. Virology. 2015;478:1-8.

25. Luo $R$, Xiao $S$, Jiang $Y$, Jin $H$, Wang $D$, Liu $M$, et al. Porcine reproductive and respiratory syndrome virus (PRRSV) suppresses interferon-beta production by interfering with the RIG-I signaling pathway. Mol Immunol. 2008:45:2839-46.
26. Fensterl V, Grotheer D, Berk I, Schlemminger S, Vallbracht A, Dotzauer A. Hepatitis A virus suppresses RIGI-mediated IRF-3 activation to block induction of beta interferon. J Virol. 2005;79:10968-77.

27. Peng T, Kotla S, Bumgarner RE, Gustin KE. Human rhinovirus attenuates the type I interferon response by disrupting activation of interferon regulatory factor 3. J Virol. 2007;81:6161.

28. Iwamura T, Yoneyama M, Yamaguchi K, Suhara W, Mori W, Shiota K, et al. Induction of IRF-3/-7 kinase and NF-kappaB in response to double-stranded RNA and virus infection: common and unique pathways. Genes Cells. 2001;6:375-88.

29. Fitzgerald KA, McWhirter SM, Faia KL, Rowe DC, Latz E, Golenbock DT, et al. IKKepsilon and TBK1 are essential components of the IRF3 signaling pathway. Nat Immunol. 2003;4:491-6.

30. Xu X, Zhang $H$, Zhang Q, Dong J, Liang $Y$, Huang $Y$, et al. Porcine epidemic diarrhea virus $E$ protein causes endoplasmic reticulum stress and upregulates interleukin-8 expression. Virol J. 2013;10:26.

31. Xu X, Zhang H, Zhang Q, Huang Y, Dong J, Liang Y, et al. Porcine epidemic diarrhea virus $\mathrm{N}$ protein prolongs $\mathrm{S}$-phase cell cycle, induces endoplasmic reticulum stress, and up-regulates interleukin-8 expression. Vet Microbiol. 2013;164:212-21.

32. Yoneyama M, Kikuchi M, Natsukawa T, Shinobu N, Imaizumi T, Miyagishi M, et al. The RNA helicase RIG-I has an essential function in double-stranded RNA-induced innate antiviral responses. Nat Immunol. 2004;5:730-7.

\section{Submit your next manuscript to BioMed Central and take full advantage of:}

- Convenient online submission

- Thorough peer review

- No space constraints or color figure charges

- Immediate publication on acceptance

- Inclusion in PubMed, CAS, Scopus and Google Scholar

- Research which is freely available for redistribution 